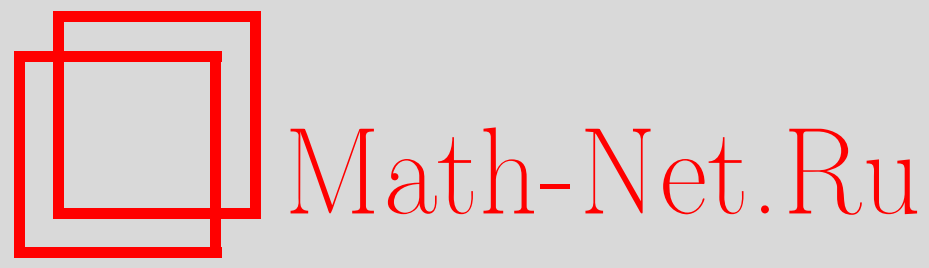

И. В. Мельникова, Свойства $d$-полугрупп Лионса и обобщенная корректность задачи Коши, Функи. анализ и его прил., 1997, том 31, выпуск 3, 23-34

DOI: https://doi.org/10.4213/faa472

Использование Общероссийского математического портала MathNet.Ru подразумевает, что вы прочитали и согласны с пользовательским соглашением

http://www . mathnet.ru/rus/agreement

Параметры загрузки:

IP : 54.209 .52 .79

26 апреля 2023 г., 14:38:20

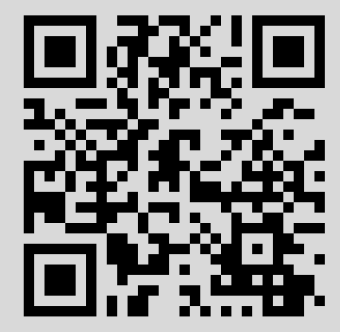




\title{
Свойства d-полугрупп Лионса и обобщенная корректность задачи Коши
}

\author{
(c) 1997. И. В. МЕЛЬНИковА
}

\section{$\S 1$. Введение}

В последнее время большой интерес, связанный с их широким применением, вызывают некорректные краевые задачи для дифференциально-операторных уравнений. Среди них особое место занимает задача Коши

$$
u^{\prime}(t)=A u(t), \quad t \geqslant 0, \quad u(0)=x, \quad A: D(A) \subset X \rightarrow X,
$$

в банаховом пространстве $X$ с оператором $A$, не являющимся генератором сильно непрерывной полугруппы. Современный подход к решению этой задачи состоит в построении различных семейств ограниченных операторов: регуляризаторов, интегрированных полугрупп, $C$-полугрупп (локальных и глобальных). $\mathrm{C}$ помощью таких полугрупп на некотором подмножестве из $D(A)\left(D\left(A^{n}\right)\right.$ для интегрированных полугрупп и $C D(A)$ для $C$-полугрупп) строится решение задачи, устойчивое относительно изменения $x$ по некоторой более сильной норме, чем норма исходного пространства (см. $[4,8,10,12,15,16]$ и др.). Обзор [2] посвящен полугрупповым методам решения задач Коши - задачи (1) и вырожденной задачи

$$
B u^{\prime}(t)=A u(t), \quad t \geqslant 0, \quad u(0)=x, \quad \text { ker } B \neq\{0\} .
$$

Регуляризаторы позволяют строить приближенное решение задачи (зависяшее от параметра погрешности начальных данных и параметра регуляризации), устойчивое в $X$. В $[3,14]$ установлена связь между полугрупповыми методами и методами регуляризации некорректной задачи Коши. Показано, что широкий класс регуляризаторов совпадает с $C$-полугруппами, зависящими от параметра регуляризации.

Настоящая работа посвящена другому подходу к решению классически некорректных задач: указаны условия на оператор $A$, при которых для любого $x$ из $X$ сушествует единственное и устойчивое решение задачи (1) в пространстве абстрактных распределений.

Распределение $U \in \mathscr{D}_{0}^{\prime}(X)$ является (обобщенным) решением задачи (1), если

$$
\left\langle U, \varphi^{\prime}\right\rangle+A\langle U, \varphi\rangle=-\langle\delta, \varphi\rangle x, \quad x \in X, \quad \forall \varphi \in \mathscr{D},
$$

или (что эквивалентно)

$$
P * U=\delta \otimes x, \quad P:=\delta^{\prime} \otimes I-\delta \otimes A \in \mathscr{D}_{0}^{\prime}\left(\mathcal{L}\left(X_{A}, X\right)\right),
$$

\footnotetext{
^ Работа выполнена при поддержке РФФИ, грант №95-01-00283.
} 
где
$\left\langle\delta^{\prime} \otimes I, \varphi\right\rangle:=\left\langle\delta^{\prime}, \varphi\right\rangle I$,
$\langle\delta \otimes A, \varphi\rangle:=\langle\delta, \varphi\rangle A$,
$\langle\delta \otimes x, \varphi\rangle:=\langle\delta, \varphi\rangle x$,

$\mathscr{D}$ - пространство основных функций, $\mathscr{D}^{\prime}(X):=\mathscr{L}(\mathscr{D}, X)$ - пространство абстрактных распределений, $\mathscr{D}_{0}^{\prime}(X)$ - подпространство распределений с носителем в $\mathbb{R}_{+}, X_{A}:=\left\{D(A),\|x\|_{A}:=\|x\|+\|A x\|\right\}$.

Для любого классического решения задачи (1) распределение $U=H(t) u(t)$ $(H(t)$ - функция Хевисайда) является решением уравнений (3), (4). Впервые понятие решения и корректности абстрактной задачи Коши в смысле распределений было введено Лионсом [13]: вместо операторов решения $T(t)(T(t) x:=$ $u(t))$, порождающих полугруппы с различными свойствами в банаховом пространстве $X$, введена полугруппа-распределение $Q \in \mathscr{D}_{0}^{\prime}(\mathscr{L}(X))$, удовлетворяющая «полугрупповому» соотношению

$$
\langle Q, \varphi * \psi\rangle=\langle Q, \varphi\rangle\langle Q, \psi\rangle, \quad \varphi, \psi \in \mathscr{D}_{0} .
$$

Доказано, что существование полугруппы-распределения эквивалентно существованию решения уравнений

$$
P * Q=\delta \otimes I, \quad Q * P=\delta \otimes J,
$$

где $I$ и $J$ - единичные операторы на $X$ и $X_{A}$ соответственно, а $A$ - генератор полугруппы. Чазарайн доказал, что существование решения уравнений (5) эквивалентно существованию единственного решения уравнения (4) [6]. В [11, 17, $18,20,9]$ дана характеристика оператора, порождающего обобщенно-корректную задачу в терминах его резольвенты (обобщенной резольвенты, асимптотической резольвенты).

Однако только после появления теории интегрированных полугрупп стала понятна суть всех этих связей и характеристик: как известно, структурные теоремы для распределения $F$ гарантируют существование $m \geqslant 0$ и непрерывной функции $f(t)$, таких, что $F=f^{(m)}$. Для абстрактных распределений эти теоремы тоже имеют место [9]. Так вот, интегрированная полугруппа $V(t)$ и есть та самая непрерывная функция, существование которой для полугруппыраспределения $Q$ гарантируют структурные теоремы. В частности, для экспоненциально ограниченной $Q$ существует экспоненциально ограниченная интегрированная полугруппа, а для $Q \in \mathscr{D}_{0}^{\prime}\left(\mathscr{L}\left(X, X_{A}\right)\right)$ существует локальная интегрированная полугруппа $[1,14]$. В $[19]$ связь между локальными интегрированными полугруппами и полугруппами-распределениями была установлена через существование эквинепрерывной полугруппы в пространстве Фреше $Y:=$ $\left\{\bigcap D\left(A^{k}\right),\|x\|_{k}=\left\|A^{k} x\right\|\right\}$.

В настоящей работе указаны условия, эквивалентные обобщенной корректности задачи Коши. Дано конструктивное доказательство связи между существованием некоторой локальной интегрированной полугруппы, существованием полугруппы-распределения и существованием локальной интегрированной полугруппы $\{V(t), 0 \leqslant t<T\}$ для любого $T<\infty$. Доказано, что существование любой из этих полугрупп эквивалентно обобщенной корректности задачи Коши с генератором полугруппы в качестве оператора $A$. 


\section{§2. Задача Коши в пространстве распределений. Свойства d-полугрупп}

Пусть $X-$ банахово пространство и $\mathscr{D}\left(\mathscr{D}_{0}, \mathscr{E}, \mathscr{E}_{0}\right)$ - пространство ШШварца бесконечно дифференцируемых функций $\varphi: \mathbb{R} \rightarrow \mathbb{R}$ с компактными носителями (с компактными носителями в $\mathbb{R}_{+}$, с произвольными носителями, с носителями в $\left.\mathbb{R}_{+}\right)$. Для значений распределений $U$ на функциях $\varphi$ из этих пространств будем пользоваться обозначениями $U(\varphi)$ или $\langle U, \varphi\rangle$. Подобно доказательству того факта, что любой линейный непрерывный оператор является ограниченным, для абстрактных распределений можно установить такой результат:

Лемма 1. Пусть $U \in \mathscr{D}^{\prime}(X)$ и $K-$ компактное множество в $\mathbb{R}$. Тогда существуют $p \geqslant 0, c>0$, такие, что

$$
\|U(\varphi)\| \leqslant c\|\varphi\|_{p}, \quad \varphi \in \mathscr{D}(\operatorname{supp} \varphi \subset K), \quad \text { əde }\|\varphi\|_{p}:=\sup _{0 \leqslant j \leqslant p, t \in K}\left|\varphi^{(j)}(t)\right| .
$$

Пусть $\mathscr{D}^{p}(K)$ - банахово пространство $p$ раз непрерывно дифференцируемых функций с носителями в компактном множестве $K$ и с нормой $\|\cdot\|_{p}$.

Лемма 2. Для любых $\varphi \in \mathscr{D}^{p}(K)$ u $\varepsilon>0$ существует функиия $\psi \in \mathscr{D}$, такая, что

$$
\operatorname{supp} \psi \subseteq K_{\varepsilon}=\{t \in \mathbb{R}: \operatorname{dist}(t, K) \leqslant \varepsilon\} \quad u \quad\|\varphi-\psi\|_{p} \leqslant \varepsilon .
$$

Эти леммы означают, что любое распределение из $\mathscr{D}^{\prime}(X)$ локально можно продолжить на пространство достаточно гладких функций. На этом основана следующая «структурная»

Teорема [9]. Пусть $U \in \mathscr{D}^{\prime}(X)$ и $\Omega$ - открытое ограниченное множество из $\mathbb{R}$. Тогда существует $m>0$ и непрерьввная функция $f: \mathbb{R} \rightarrow X$, такие, что для любой функиии $\varphi \in \mathscr{D}$ с носителем в $\Omega$

$$
U(\varphi)=f^{(m)}(\varphi) .
$$

Если $U=0$ на $(-\infty, a)$, то $f(t)=0$ для $t<a$.

На основе этой теоремы определяется операция свертки, необходимая для постановки обобщенной задачи Коши. Пусть $X, Y$ - банаховы пространства; тогда для $U \in \mathscr{D}_{0}^{\prime}(\mathscr{L}(X, Y)), V \in \mathscr{D}_{0}^{\prime}(X)$

$$
\langle(U * V), \varphi\rangle:=\left\langle(f * g)^{(m+p)}, \varphi\right\rangle,
$$

где функции $f$ и $g$ определяются структурной теоремой: $U(\varphi)=f^{(m)}(\varphi)$, $V(\varphi)=g^{(p)}(\varphi)$. При этом свертка $U * V \in \mathscr{D}_{0}^{\prime}(Y)$ не зависит от выбора $m$, $p, f, g$.

Пусть $A$ - линейный замкнутый плотно определенный в банаховом пространстве $X$ оператор.

ОПРЕДЕЛЕНИЕ 1. $U \in \mathscr{D}_{0}^{\prime}\left(X_{A}\right)$ называется решением задачи Коши (1) в смысле распределений (обобшенным решением), если оно является решением уравнения (4). Задача Коши называется корректной в смысле распределений (обобщенно корректной), если для любого $x \in X$ существует единственное решение уравнения (4) и для любой последовательности $x_{n} \rightarrow 0$ соответствующие $U_{n}$ сходятся к 0 в пространстве $\mathscr{D}_{0}^{\prime}\left(X_{A}\right)$. 
Нетрудно проверить, что если $u(t)$ - классическое решение задачи (1), то распределение

$$
U(\varphi):=\int_{0}^{\infty} \varphi(t) u(t) d t, \quad \varphi \in \mathscr{D},
$$

является решением уравнения (4). Действительно,

$$
\begin{aligned}
& \int_{0}^{\infty} \varphi(t) u^{\prime}(t) d t=-\varphi(0) x-\int_{0}^{\infty} \varphi^{\prime}(t) u(t) d t=-(\delta \otimes x)(\varphi)+U^{\prime}(\varphi), \\
& \int_{0}^{\infty} \varphi(t) u^{\prime}(t) d t=A \int_{0}^{\infty} \varphi(t) u(t) d t=A U(\varphi) .
\end{aligned}
$$

Учитывая определение оператора $P$, получим

$$
\begin{aligned}
\langle P * U, \varphi\rangle & =\left\langle\left(\delta^{\prime} \otimes I\right) * U, \varphi\right\rangle-\langle(\delta \otimes A) * U, \varphi\rangle \\
& =\left\langle U^{\prime}, \varphi\right\rangle-\langle A U, \varphi\rangle=\langle\delta \otimes x, \varphi\rangle .
\end{aligned}
$$

Подобно случаю равномерно корректной задачи Коши, решение обобщеннокорректной задачи Коши связано с полугруппой операторов решения. В случае уравнения (4) это полугруппа-распределение, введенная Лионсом.

ОПРЕДЕЛЕНИЕ 2. Назовем распределение $Q \in \mathscr{D}_{0}^{\prime}(\mathscr{L}(X))$ nолугруппой-распределением (d-полугруппой), если

(i) $\langle Q, \varphi * \psi\rangle=\langle Q, \varphi\rangle\langle Q, \psi\rangle$ для любых $\varphi, \psi \in \mathscr{D}_{0}$;

(ii) для любой функции $\varphi \in \mathscr{D}_{0} \quad Q(\varphi) x=0 \Longrightarrow x=0$;

d-полугруппа называется регулярной, если

(iii) для любого $y=Q(\varphi) x\left(\varphi \in \mathscr{D}_{0}, x \in X\right)$ распределение $Q y$ - это непрерывная функция $f(t)$, такая, что $f(t)=0$ для $t<0$ и $f(0)=y$;

d-полугруппа имеет плотную область значений, если

(iv) множество $\left\{Q(\varphi) x: x \in X, \varphi \in \mathscr{D}_{0}\right\}$ плотно в $X$.

В [13] d-полугруппой названо распределение $Q \in \mathscr{D}_{0}^{\prime}(\mathscr{L}(X))$, удовлетворяющее условиям (i)-(iv).

Генератор $A$ d-полугруппы определяется следующим образом: $A:=\overline{Q\left(-\delta^{\prime}\right)}$. Чтобы обосновать это определение, для распределений z с компактным носителем на полуоси определяется оператор $Q(z):$ пусть $z \in \mathscr{E}_{0}^{\prime}\left(\mathscr{E}_{0}^{\prime}-\right.$ подпространство распределений из $\mathscr{E}$ с носителями в $\left.\mathbb{R}_{+}\right)$и $\delta_{n} \in \mathscr{D}_{0}-$ произвольная $\delta$-образная последовательность, тогда

$D(Q(z)):=\left\{x: Q\left(\delta_{n}\right) x \rightarrow x\right.$ и существует $y$, такой, что $\left.Q\left(z * \delta_{n}\right) x \rightarrow y\right\}$,

$$
Q(z) x:=y \text {. }
$$

Установим свойства введенных таким образом d-полугрупп. На основе этих свойств в основной теореме будет доказано, что распределение $U:=Q x$ является единственным обобщенным решением задачи (1).

ПРЕДЛОЖЕНИЕ 1. Пусть $z \in \mathscr{E}_{0}^{\prime} u Q$ есть d-полугруппа с плотной областью значений. Тогда $Q(z)$ обладает следуюшими свойствами:

(Q1) $Q(\varphi) x \in D(Q(z))$ u $Q(z) Q(\varphi) x=Q(z * \varphi) x$ для любих $\varphi \in \mathscr{D}_{0}$, $x \in X$; 
(Q2) $\overline{D(Q(z))}=X$;

(Q3) $Q(z) Q(\varphi) x=Q(\varphi) Q(z) x$ для любих $x \in D(Q(z)), \varphi \in \mathscr{D}_{0}$;

(Q4) существует замыкание $\overline{Q(z)}$ оператора $Q(z)$ и $\overline{Q(z)} Q(\varphi) x=$ $Q(z) Q(\varphi) x$ для любих $\varphi \in \mathscr{D}_{0}, x \in X$;

Если $Q$ - регулярная d-полугруппа, то

(Q5) $\overline{Q\left(\psi_{+}\right)}=Q(\psi)$ для любого $\psi \in \mathscr{D}$, где $\psi_{+}(t)= \begin{cases}\psi(t), & t \geqslant 0, \\ 0, & t<0,\end{cases}$ $\psi_{+}(t) \in \mathscr{E}_{0}$.

ДокАЗАТЕльСтво. (Q1) В силу того, что $\delta_{n} * \varphi \in \mathscr{D}_{0}$, и в силу свойства (i) имеем

$$
\begin{aligned}
Q\left(\delta_{n}\right) Q(\varphi) x & =Q\left(\delta_{n} * \varphi\right) x \rightarrow Q(\varphi) x, \\
Q\left(z * \delta_{n}\right) Q(\varphi) x & =Q\left(z * \delta_{n} * \varphi\right) x \rightarrow Q(z * \varphi) x, \quad x \in X .
\end{aligned}
$$

Отсюда $Q(\varphi) x \in D(Q(z))$ и $Q(z) Q(\varphi) x=Q(z * \varphi) x$.

Из (Q1) и свойства (iv) следует (Q2).

(Q3) Для $x, y$ из определения (11) имеем

$$
\begin{gathered}
Q(\varphi) Q\left(z * \delta_{n}\right) x \rightarrow Q(\varphi) y=Q(\varphi) Q(z) x \\
Q(\varphi) Q\left(z * \delta_{n}\right) x=Q\left(z * \delta_{n} * \varphi\right) x \rightarrow Q(z * \varphi) x=Q(z) Q(\varphi) x .
\end{gathered}
$$

(Q4) Известно, что линейный оператор $A$ имеет замыкание, если и только если для любой последовательности $x_{n} \in D(A)$ из того, что $x_{n} \rightarrow 0, A x_{n} \rightarrow y$, следует, что $y=0$.

Пусть $x_{n} \in D(Q(z)), x_{n} \rightarrow 0, Q(z) x_{n} \rightarrow y$; тогда

$$
Q(\varphi) Q(z) x_{n}=Q(z * \varphi) x_{n} \rightarrow Q(\varphi) y, \quad \varphi \in \mathscr{D}_{0} ;
$$

отсюда и из того, что $Q(z * \varphi) \in \mathscr{L}(X)$, получаем $Q(\varphi) y=0$. Из (iі) следует $y=0$.

(Q5) Пусть $\psi \in \mathscr{D}$; в силу (Q4) для $\psi_{+} \in \mathscr{E}_{0}$ имеем

$$
\overline{Q\left(\psi_{+}\right)} Q(\varphi) x=Q\left(\psi_{+}\right) Q(\varphi) x=Q\left(\psi_{+} * \varphi\right) x, \quad \varphi \in \mathscr{D}_{0}, x \in X .
$$

Если мы докажем, что $Q\left(\psi_{+}\right) Q(\varphi) x=Q(\psi) Q(\varphi) x$, то с учетом (iv) будет доказано (Q5). В силу регулярности d-полугруппы $Q$ существует непрерывная функция $f(t) \in C(\mathbb{R}, X)(f(t)=0, t<0)$, такая, что

$$
\begin{aligned}
Q\left(\psi_{+}\right) y & =\lim _{n \rightarrow \infty} Q\left(\psi_{+} * \delta_{n}\right) y=\lim _{n \rightarrow \infty} \int_{0}^{\infty} f(t) d t \int_{0}^{\infty} \psi(\tau) \delta_{n}(t-\tau) y d \tau \\
& =\int_{0}^{\infty} f(t) \psi(t) y d t=Q(\psi) y \quad \forall y=Q(\varphi) x, x \in X .
\end{aligned}
$$

\section{$\S 3$. Условия, эквивалентные обобщенной корректности задачи Коши}

ОПРЕДЕЛЕНИЕ 3 [19]. Пусть $X-$ банахово пространство, $n \in \mathbb{N}, T<\infty$. Однопараметрическое семейство $\{V(t), 0 \leqslant t<T\}$ ограниченных в $X$ операторов называется локальной $n$ раз интегрированной полугруппой, если выполняются соотношения 
(V1) $\frac{1}{(n-1) !} \int_{0}^{s}\left[(s-r)^{n-1} V(t+r)-(t+s-r)^{n-1} V(r)\right] d r=V(t) V(s)$, $s, t, t+s \in[0, T), V(0)=0$;

(V2) $V(t)$ сильно непрерывна по $t \in[0, T)$;

(V3) $\forall t \in[0, T) \quad V(t) x=0 \Longrightarrow x=0$.

Генератор полугруппы $V(t)$ определяется как замыкание оператора

$$
A_{0} x:=\lim _{t \rightarrow 0} t^{-1}\left[V^{(n)}(t) x-x\right], \quad D\left(A_{0}\right)=\left\{x \in X: \exists \lim _{t \rightarrow 0} t^{-1}\left[V^{(n)}(t) x-x\right]\right\} .
$$

ОСНОВНАЯ теОРемА. Пусть $A-$ - инейный замкнутый плотно определенный в $X$ оператор. Тогда следующие утверждения эквивалентнь:

(A) Задача Коши (1) является обобщенно корректной;

(В) A является генератором регулярной d-полугруппь с плотной областью значений;

(C) A является генератором локальной интегрированной полугруппь;

(C1) для любого $T>0$ оператор $A$ является генератором локальной $n$ раз интегрированной полугруппь $\{V(t), t \in[0, T)\}(n=n(T))$;

(D) существует область

$$
\Lambda:=\left\{\lambda \in \mathbb{C}: \operatorname{Re} \lambda>\frac{k}{\tau} \ln (1+|\lambda|)+\frac{1}{\tau} \ln \frac{c}{\gamma}\right\} \subset \rho(A)
$$

$(\tau \in(0, T), c>0,0<\gamma<1, k=k(n))$, такая, ито для некоторого $C$

$$
\left\|R_{A}(\lambda)\right\| \leqslant C|\lambda|^{k} / \operatorname{Re} \lambda, \quad \lambda \in \Lambda .
$$

ДокаЗАтЕльство. 1. (А) $\Longleftrightarrow(\mathrm{B})$. Покажем, что из утверждения (В) следует, что

существует $Q \in \mathscr{D}_{0}^{\prime}\left(\mathscr{L}\left(X, X_{A}\right)\right)$, такое, что $P * Q=\delta \otimes I, Q * P=\delta \otimes J$.

Отсюда, учитывая доказанную в [6] эквивалентность существования единственного решения уравнения (4) и существования решения уравнений (5), получим обобщенную корректность задачи (1). Пусть $Q$ есть d-полугруппа с плотной областью значений. Для $\psi \in \mathscr{D}$ и функции $\psi_{+}$, введенной в (Q5) предложения 1 , имеем $\psi_{+} \in \mathscr{E}_{0}^{\prime}$ и

$$
\delta^{\prime} * \psi_{+}=\psi_{+}^{\prime}+\psi(0) \delta .
$$

В силу свойства (Q1) и определения генератора d-полугруппы

$$
\begin{aligned}
Q\left(\delta^{\prime} * \psi_{+} * \varphi\right) x & =Q\left(\psi_{+}^{\prime} * \varphi\right) x+\psi(0) Q(\varphi) x, \\
Q\left(\delta^{\prime}\right) Q\left(\psi_{+} * \varphi\right) x & =-A Q\left(\psi_{+} * \varphi\right) x=-A Q\left(\psi_{+}\right) Q(\varphi) x=Q\left(\psi_{+}\right) Q\left(\delta^{\prime} * \varphi\right) x \\
& =Q\left(\psi_{+}\right)(-A) Q(\varphi) x, \quad \varphi \in \mathscr{D}_{0}, x \in X, .
\end{aligned}
$$

Отсюда в силу свойства (Q5)

$$
-A Q(\psi) Q(\varphi) x=Q\left(\psi^{\prime}\right) Q(\varphi) x+\psi(0) Q(\varphi) x=-Q(\psi) A Q(\varphi) x,
$$

т. е. для $y=Q(\varphi) x$ имеют место равенства

$$
\begin{aligned}
& -A Q(\psi) y-Q\left(\psi^{\prime}\right) y=\psi(0) y, \\
& -Q(\psi) A y-Q\left(\psi^{\prime}\right) y=\psi(0) y .
\end{aligned}
$$


Из того, что область значений d-полугруппы плотна в $X$, а оператор $A$ является замкнутым, следует, что $Q(\psi) y \in D(A)$ и (8) выполняется для любого $y \in X$. Пусть $\left\|y_{n}\right\| \rightarrow 0$ в равенстве (8); тогда $\left\|A Q(\psi) y_{n}\right\| \rightarrow 0$, а следовательно, $Q(\psi) \in \mathscr{L}\left(X, X_{A}\right)$. Если в (8) $\psi_{n} \rightarrow 0\left(\psi_{n} \in \mathscr{D}\right)$, то $\left\|Q\left(\psi_{n}^{\prime}\right)\right\|$, $\left\|A Q\left(\psi_{n}\right)\right\| \rightarrow 0 ;$ значит, $Q \in \mathscr{D}^{\prime}\left(\mathscr{L}\left(X, X_{A}\right)\right)$. Учитывая, что $\operatorname{supp} Q \in[0, \infty)$, имеем $Q \in \mathscr{D}_{0}^{\prime}\left(\mathscr{L}\left(X, X_{A}\right)\right)$. Чтобы завершить доказательство, остается показать, что уравнение (9) выполняется для любого $y \in D(A)$. Положим в $(7)$ $x \in D\left(Q\left(-\delta^{\prime}\right)\right), \varphi_{n} \rightarrow \delta$. Тогда

$$
Q\left(\varphi_{n}\right) x \rightarrow x, \quad Q\left(\delta^{\prime} * \varphi_{n}\right) x \rightarrow-A x ;
$$

отсюда вытекает, что

$$
-Q(\psi) A x=Q\left(\psi^{\prime}\right) x+\psi(0) x, \quad x \in D\left(Q\left(-\delta^{\prime}\right)\right) .
$$

Из определения генератора d-полугруппы следует, что для любого $y \in D(A)$ существуют $x_{n} \in D\left(Q\left(-\delta^{\prime}\right)\right)$, такие, что

$$
x_{n} \rightarrow y, \quad A x_{n} \rightarrow A y
$$

отсюда вытекает, что уравнение (10) имеет место для любого $x \in D(A)$, т. е. (9) имеет место для любого $y \in D(A)$. Итак, d-полугруппа $Q$ является решением уравнений (5), а значит, $U:=Q x$ является единственным решением уравнения $(4)[6,1]$. Из ограниченности оператора $Q(\varphi)$ следует устойчивость решения: $x_{n} \rightarrow x \Longrightarrow U_{n} \rightarrow U$ в пространстве $\mathscr{D}_{0}^{\prime}\left(X_{A}\right)$. Обратно, из корректности задачи Коши следует существование решения уравнений $(5)\langle Q, \varphi\rangle x:=\langle U, \varphi\rangle$. Лионсом [13] доказано, что решение уравнений (5) является регулярной невырожденной полугруппой с плотной областью значений.

2. $(\mathrm{A}) \Longleftrightarrow(\mathrm{C}) \Longleftrightarrow(\mathrm{C} 1) \Longleftrightarrow(\mathrm{D})$. Сначала докажем $(\mathrm{C}) \Longrightarrow(\mathrm{D}) \Longrightarrow(6)$, a затем $(6) \Longrightarrow(\mathrm{C} 1)$.

Пусть $A$ - генератор $n$ раз локальной интегрированной полугруппы $V(t)$, $0 \leqslant t<T$. Тогда для $\tau \in(0, T)$ определена ограниченная оператор-функция $R(\lambda, \tau)$ - «локальная резольвента» оператора $A$ :

$$
R(\lambda, \tau) x:=\int_{0}^{\tau} \lambda^{n} e^{-\lambda t} V(t) x d t, \quad x \in X .
$$

Для $x \in D(A)$ применим оператор $\lambda-A$ к равенству (11). Учитывая уравнение, которому удовлетворяет $V(t)$,

$$
V(t) x=\frac{t^{n}}{n !} x+\int_{0}^{t} V(s) A x d s, \quad x \in D(A), t \in(0, T),
$$

и перестановочность $V(t)$ и $A$ на $D(A)$ (см. $[19,2]$ ), интегрируя по частям, получаем равенства

$$
\begin{aligned}
(\lambda-A) R(\lambda, \tau) x & =\int_{0}^{\tau} \lambda^{n+1} e^{-\lambda t} V(t) x d t-\int_{0}^{\tau} \lambda^{n} e^{-\lambda t} A V(t) x d t \\
& =-\lambda^{n} e^{-\lambda \tau} V(\tau) x+\frac{(\lambda \tau)^{n}}{n !} e^{-\lambda \tau} x+\int_{0}^{\tau} \frac{t^{n}}{n !} e^{-\lambda t} d t x \\
& \equiv(I-G(\lambda)) x, \quad x \in D(A), \\
R(\lambda, \tau)(\lambda-A) x & =(I-G(\lambda)) x, \quad x \in D(A),
\end{aligned}
$$


где

$$
G(\lambda) x=\lambda^{n} e^{-\lambda \tau} V(\tau) x+\sum_{k=0}^{n-1} \frac{(\lambda \tau)^{k}}{k !} e^{-\lambda \tau} x
$$

и $G$ - ограниченный оператор:

$$
\|G(\lambda) x\| \leqslant c(1+|\lambda|)^{n} e^{-\tau \operatorname{Re} \lambda}\|x\|, \quad c=c(\tau, n) .
$$

В силу ограниченности оператора $G$, замкнутости $A$ и плотности множества $D(A)$ равенство (13) можно продолжить на все пространство $X$. Используя полученную для $G$ оценку, найдем область $\Lambda \subset \mathbb{C}$, в которой $\|G(\lambda)\|<1$. Прологарифмировав неравенство $c(1+|\lambda|)^{n} e^{-\tau \operatorname{Re} \lambda}<\gamma<1$, получим, что в области

$$
\Lambda:=\left\{\lambda \in \mathbb{C}: \operatorname{Re} \lambda>\frac{n}{\tau} \ln (1+|\lambda|)+\frac{1}{\tau} \ln \frac{c}{\gamma}\right\}
$$

имеют место оценки

$$
\|G(\lambda)\|<\gamma, \quad\left\|(I-G(\lambda))^{-1}\right\|<1 /(1-\gamma) .
$$

Отсюда для $\lambda \in \Lambda$ следует существование ограниченного оператора

$$
(\lambda-A)^{-1}=R(\lambda, \tau)(I-G(\lambda))^{-1},
$$

для которого

$$
\begin{aligned}
\left\|(\lambda-A)^{-1}\right\| & \leqslant \frac{1}{1-\gamma} \int_{0}^{\tau}|\lambda|^{n} e^{-t \operatorname{Re} \lambda}\|V(t)\| d t \\
& \leqslant \sup _{0 \leqslant t \leqslant T}\|V(t)\| \frac{|\lambda|^{n}\left(1-e^{-\tau \operatorname{Re} \lambda}\right)}{(1-\gamma) \operatorname{Re} \lambda} \leqslant \frac{C|\lambda|^{n}}{\operatorname{Re} \lambda},
\end{aligned}
$$

т.е. выполняется (D): существует $C$, такое, что

$$
\left\|R_{A}(\lambda)\right\| \leqslant \frac{C|\lambda|^{n}}{\operatorname{Re} \lambda}, \quad \lambda \subset \Lambda .
$$

Теперь по резольвенте, удовлетворяющей условию (14), построим решение уравнений (5). Для любого $p \geqslant 2 n+2$ определим функцию

$$
T_{p}(t):=\frac{1}{2 \pi i} \int_{\partial \Lambda} \lambda^{-p} e^{\lambda t} R_{A}(\lambda) d \lambda .
$$

Функция под интегралом растет не быстрее, чем $|\lambda|^{n t / \tau+n-p} ;$ следовательно, интеграл определяет непрерывную по $t \in\left(-\infty, \tau_{p}\right)$, где $\tau_{p}=\tau(p-n-1) / n$, функцию со значениями в пространстве $\mathscr{L}\left(X, X_{A}\right)$.

Положим $Q=T_{p}^{(p)}$ на $\left(-\infty, \tau_{p}\right)$. При таком определении $Q$ не зависит от $p$; поэтому для любой функции $\varphi(\operatorname{supp} \varphi \in(-\infty, \tau))$ можно выбрать $\tau_{p}$ равным $\tau$ за счет увеличения $p$ и $\langle Q, \varphi\rangle=\left\langle T_{p}^{(p)}, \varphi\right\rangle$. В силу абстрактной теоремы Коши $T_{p}(t)=0, t \leqslant 0$. 
Покажем, что $Q$ удовлетворяет уравнениям (5):

$$
\begin{aligned}
&\langle Q * P, \varphi\rangle x= Q^{\prime}(\varphi) x-Q(\varphi) A x \\
&= \frac{(-1)^{p+1}}{2 \pi i} \int_{0}^{\tau_{p}} \varphi^{(p+1)}(t) \int_{\partial \Lambda} \lambda^{-p} e^{\lambda t} R_{A}(\lambda) x d \lambda d t \\
& \quad-\frac{(-1)^{p}}{2 \pi i} \int_{0}^{\tau_{p}} \varphi^{(p)}(t) \int_{\partial \Lambda} \lambda^{-p} e^{\lambda t} R_{A}(\lambda) A x d \lambda d t \\
&= \frac{(-1)^{p}}{2 \pi i} \int_{0}^{\tau_{p}} \varphi^{(p)}(t) \int_{\partial \Lambda} \lambda^{-p} e^{\lambda t} x d \lambda d t, \\
& \varphi \in \mathscr{D}, \operatorname{supp} \varphi \subset\left(-\infty, \tau_{p}\right), x \in D(A) .
\end{aligned}
$$

Отсюда, пользуясь абстрактной формулой Коши для внутреннего интеграла, получаем

$$
\begin{aligned}
\langle Q * P, \varphi\rangle x & =(-1)^{p} \int_{0}^{\tau_{p}} \varphi^{(p)}(t) \frac{t^{p-1}}{(p-1) !} d t x \\
& =\varphi(0) x=\langle\delta \otimes x, \varphi\rangle=\langle\delta \otimes J, \varphi\rangle x .
\end{aligned}
$$

Аналогично доказывается выполнение второго из уравнений (5). Таким образом, дано построение распределения $Q \in \mathscr{D}_{0}^{\prime}\left(\mathscr{L}\left(X, X_{A}\right)\right)$ в импликации $(\mathrm{D}) \Longrightarrow(6)$.

Пусть теперь $Q$ - решение уравнений (5). Положим $K=[-1, T]$. С учетом лемм 1,2 по распределению $Q$ (обратному к $P$ ) строится его продолжение на пространство $\mathscr{D}^{p}(K)$ и принадлежащая $\mathscr{D}^{p}(K)$ функция

$$
\psi_{t, p}(s)=: \chi(s) \eta(t-s), \quad t \in[0, T],
$$

где $\chi(s)$ - произвольная бесконечно дифференцируемая функция, равная нулю при $s \leqslant-1$ и единице при $s \geqslant 0$, а $\eta(s)$ - непрерывная функция с носителем в $[0, \infty)$, такая, что $\eta^{(p+2)}=\delta$. Локальная $p+2$ раза интегрированная полугруппа $\{V(t), 0 \leqslant t<T\}$ строится, подобно первообразной порядка $p+2$ в структурной теореме:

$$
V(t):=Q\left(\psi_{t, p}\right) .
$$

Построенная таким образом $V(t)$ - это сильно непрерывная по $t$ операторфункция со свойствами $V(t) \in \mathscr{L}(X)$ при каждом $t, V(0)=0$ и (V3) из определения 3. Покажем выполнение «полугруппового» соотношения (V1). В силу леммы 2 существует последовательность $\left\{\varphi_{n}\right\} \in \mathscr{D}$, такая, что

$$
\operatorname{supp} \varphi_{n} \in[-1-\varepsilon, T+\varepsilon], \quad\left\|\varphi_{n}-\psi_{t, p+1}\right\|_{p+1} \rightarrow 0, \quad t \in[0, T] ;
$$

следовательно,

$$
\left\|\varphi_{n}-\psi_{t, p+1}\right\|_{p},\left\|\varphi_{n}^{\prime}-\psi_{t, p+1}^{\prime}\right\|_{p} \rightarrow 0 .
$$

Рассмотрим уравнения (5) с $\varphi=\varphi_{n}$. Устремляя $n \mathrm{~K} \infty$, получим

$$
\begin{array}{ll}
-\left\langle Q, \psi_{t, p+1}^{\prime}\right\rangle x-A\left\langle Q, \psi_{t, p+1}\right\rangle x=\psi_{t, p+1}(0) x, & x \in X, \\
-\left\langle Q, \psi_{t, p+1}^{\prime}\right\rangle x-\left\langle Q, \psi_{t, p+1}\right\rangle A x=\psi_{t, p+1}(0) x, & x \in D(A) .
\end{array}
$$


В силу того что

$$
\psi_{t, p+1}^{\prime}=\chi^{\prime}(s) \frac{(t-s)^{p+2}}{(p+2) !}+\chi(s) \eta_{p}(t-s) \quad \text { и } \quad \operatorname{supp} \chi^{\prime}(s) \frac{(t-s)^{p+2}}{(p+2) !} \subset[-1,0],
$$

для $V(t)$, определенной равенством (15), имеем

$$
V(t)=\left\langle Q, \psi_{t, p}\right\rangle=-\left\langle Q, \psi_{t, p+1}^{\prime}\right\rangle=\frac{d}{d t}\left\langle Q, \psi_{t, p+1}\right\rangle \quad \text { и } \quad Q\left(\psi_{t, p+1}\right)=\int_{0}^{t} V(t) d t
$$

для любого $t \in[0, T]$. Отсюда и из уравнений $(16),(17)$ следует, что $V(t)$ удовлетворяет уравнению

$$
V(t) x=\frac{t^{p+2}}{(p+2) !}+\int_{0}^{t} V(s) A x d s, \quad x \in D(A), t \in[0, T],
$$

т. е. уравнению $n=p+2$ раз интегрированной полугруппы. Отсюда, используя дифференцируемость $V(t)$ на $D(A)$ и полугрупповое соотношение для $V^{(n)}(t)$ на $D\left(A^{n}\right)$, получаем соотношение $(\mathrm{V} 1)$ из определения 3.

\section{§4. Примеры, замечания}

Приведем примеры операторов $A$, являющихся генераторами $k$ раз интегрированных полугрупп в некоторых банаховых пространствах $X$ и, как следствие, примеры задач Коши (1), являющихся корректными в обобщенном смысле на $X$ и $k$-корректными на $D\left(A^{k+1}\right)$, т.е.

$$
\sup _{t \in[0, T]}\|u(t)\|=\sup _{t \in[0, T]}\left\|V^{(k)}(t) x\right\| \leqslant C\|x\|_{A^{k}}, \quad x \in D\left(A^{k+1}\right) \quad[2,10,19] .
$$

1. $A x:=\left(\begin{array}{cc}-h & -f \\ 0 & -h\end{array}\right) x$,

$$
D(A)=\left\{x=\left(\begin{array}{l}
x_{1} \\
x_{2}
\end{array}\right) \in L_{p}(\mathbb{R}) \times L_{p}(\mathbb{R}): h x_{1}+f x_{2}, h x_{2} \in L_{p}(\mathbb{R})\right\},
$$

где $h(s)=1+s^{2}, f(s)=s^{2 \gamma}, \gamma>0$, в пространстве $X:=\left\{L_{p}(\mathbb{R}) \times L_{p}(\mathbb{R})\right.$, $\left.\|x\|_{X}=\left\|x_{1}\right\|_{p}+\left\|x_{2}\right\|_{p}\right\}$. Для оператора решения $U(t) x=e^{-t h}\left(\begin{array}{cc}1 & -t f \\ 0 & 1\end{array}\right) x$ имеют место оценки

$$
\|U(t)\|=\max _{s \in \mathbb{R}}\left[t\left|s^{2 \gamma}\right| e^{-t\left(1+s^{2}\right)}\right]=\gamma^{\gamma} t^{1-\gamma} e^{-t(1+\gamma / t)}=O\left(t^{1-\gamma}\right), \quad t \rightarrow 0 .
$$

При $\gamma \leqslant 1$ семейство операторов $U(t)$ образует в $X$ сильно непрерывную полугруппу с генератором $A$. При $1<\gamma \leqslant 2$ семейство операторов

$$
V(t) x=\int_{0}^{t} U(s) x d s=\frac{1}{h}\left(\begin{array}{cc}
1-e^{-h t} & t f e^{-h t}+\left(e^{-h t}-1\right) f / h \\
0 & 1-e^{-h t}
\end{array}\right) x
$$

удовлетворяет условиям (V1)-(V3) и образует 1 раз интегрированную полугруппу. Полугруппа-распределение в этом случае представляет собой регуляризацию расходящегося интеграла от оператор-функции $U(t)$, имеющей особенность в нуле. 
2. $A x:=\left\{a_{m} x_{m}\right\}_{m=1}^{\infty}, a_{m}=m / T+i\left(\exp ^{2}(m) / m^{2}-m^{2} / T^{2}\right)^{1 / 2}, D(A)=\{x \in$ $\left.l_{2}: A x \in l_{2}\right\}$, является генератором локальной $(t<n T) n$ раз интегрированной полугруппы в пространстве $X=l_{2}$

$$
\begin{aligned}
V(t) x & =\left\{\int_{0}^{\infty} \frac{(t-s)^{n-1}}{(n-1) !} e^{a_{m} s} x_{m} d s\right\}_{m=1}^{\infty} \\
& =\left\{\left[a_{m}^{-n} e^{a_{m} t}-\sum_{p=1}^{n}\left(a_{m}\right)^{-p} \frac{t^{n-p}}{(n-p) !}\right] x_{m}\right\} .
\end{aligned}
$$

Тот же оператор $A$ с $a_{m}=m+i\left(\exp ^{2}\left(m^{2}\right)-m^{2}\right)^{1 / 2}$ является генератором глобальной (но не экспоненциально ограниченной) полугруппы в $X=l_{2}$.

3. $A:=i \Delta$ является в $X=L_{p}(Q), Q \subset \mathbb{R}^{N}$, генератором $n$ раз интегрированной группы при $n>N|1 / 2-1 / p| / 2$ [5]. Для уравнения Шрёдингера с оператором $i \partial^{2} / \partial x^{2}$ операторы решения образуют сильно непрерывную полугруппу в пространстве $L_{2}(\mathbb{R})$ и только интегрированную полугруппу в пространстве $L_{p}(\mathbb{R}), p \neq 2[10]$.

ЗАмЕчАНИЕ 1 относительно связи между параметрами $k$ в утверждении (D) и $n$ в утверждении $(\mathrm{C} 1)$. В доказательстве теоремы из существования $n$ раз интегрированной полугруппы $\{V(t), 0 \leqslant t<T\}$ получена оценка на резольвенту генератора (14) с $k=n$, а из оценки (14) получено существование непрерывной функции $T_{p}(t)$, являющейся первообразной от d-полугруппы $Q$ лишь порядка $p \geqslant 2 n+2$.

ЗАмЕчАНИЕ 2. В примере 1 полугруппа операторов решения задачи (1) имеет в нуле особенность порядка $\alpha=\gamma-1$. Как следует из основной теоремы, эта полугруппа может быть «регуляризована» с помощью полугруппы-распределения. В работе [7] рассмотрена $K$-сверточная задача Коши

$$
d v(t) / d t=A v(t)+K(t) x, \quad v(0)=0, x \in X,
$$

ассоциированная с задачей (1). Решение этой задачи $v(t)=(u * K)(t)$ названо $K(t)$-обобщенным решением задачи (1). Уравнение (18) включает в себя как частный случай уравнение для интегрированных полугрупп и $C$-полугрупп. В [7] показано, что для некоторого класса полугрупп с особенностью в нуле более высокого порядка, чем степенной, регуляризация может быть осуществлена с помощью ультрараспределений. Для генераторов таких полугрупп положительная полуось должна состоять из регулярных точек. В рассматриваемый класс уравнений не входят уравнения типа обратной теплопроводности. Задача Коши для таких уравнений имеет обобщенные решения в пространствах новых обобщенных функций, введенных В. К. Ивановым [3].

\section{ЛитеРАТУРА}

1. Мельникова И. В., Альшанский М. А. Обобщенная корректность задачи Коши и интегрированные полугруппы. Докл. РАН, 343, №4, 448-451 (1995). 
2. Мельникова И. В., Альшанский М. А. Корректность задачи Коши в банаховом пространстве: регулярный и вырожденный случаи. Итоги науки и техники. Соврем. матем. и ее прил. Анализ-9, ВИНИТИ, 27, (1995), с. 5-64.

3. Мельникова И. В., Филинков А. И. Интегрированные и $C$-полугруппы. Корректность и регуляризация дифференциально-операторных задач. УМН, $\mathbf{4 9}$, №6, 111-150 (1994).

4. Arendt $W$. Vector valued Laplace transforms and Cauchy problems. Israel J. Math., 59, 327-352 (1987).

5. Arendt $W$. Sobolev Imbeddings and Integrated Semigroups. Lect. Notes Pure Appl. Math., 135, Marsel Dekker, New York, 1991.

6. Chazarain J. Problemes de Cauchy abstraits et applications a quelques problems mixtes. J. Funct. Anal., 7, 386-446 (1971).

7. Cioranescu I., Lumer G. Regularizations of evolution equations via kernels $K(t)$, $K$-evolution operators and convoluted semigroups, generation theorems. LSU Seminar Notes in Funct. Anal. and PDES 1993-1994, Louisiana State Univ., Baton Rouge, (1994), p. 45-52.

8. Davies E. B., Pang M. M. The Cauchy problem and a generalization of the HilleYosida theorem. Proc. London Math. Soc., 55, 181-208 (1987).

9. Fattorini H. O. The Cauchy Problem. Reading. Addison-Wesley, Mass., 1983.

10. Kellerman H., Hieber M. Integrated semigroups. J. Funct. Anal., 84, 160-180 (1989).

11. Komura T. Semigroups of operators in locally convex spaces. J. Funct. Anal., 2, 258-296 (1968).

12. de Laubenfels $R$. $C$-semigroups and the Cauchy problem. J. Funct. Anal., 111, 44-61 (1993).

13. Lions J.-L. Les semi-groupes distributions. Portug. Math., 19, No. 3-4, 141-161 (1960).

14. Melnikova I. V. General theory of ill-posed Cauchy problem. J. Inverse Ill-Posed Probl., 3, No. 2, 149-171 (1995).

15. Miyadera I. C-semigroups and semigroups of linear operators. Semigroup forum, 133-143 (1990).

16. Neubrander $F$. Integrated semigroups and their application to the abstract Cauchy problem. Pacif. J. Math., 135, 111-157 (1988).

17. Oharu S. Semigroups of linear operators in a Banach space. Publ. Res. Inst. Math. Sci., 27, 205-260 (1971).

18. Sova $M$. Concerning the characterization of generators of distribution semigroups. Cas. Pestov. Mat., 105, No. 4, 329-340 (1980).

19. Tanaka $N$., Okazawa $N$. Local $C$-semigroups and local integrated semigroups. Proc. London Math. Soc., 61, No. 3, 63-90 (1990).

20. Ushijima T. Some properties of regular distribution semigroups. Proc. Jpn. Acad., 45, 224-227 (1969).

Уральский государственный университет, математико-механический факультет

Поступило в редакцию 9 ноября 1995 г. 